\title{
Prevalencia de depresión en el adulto mayor que asiste al Centro Municipal Gerontológico, 2013
}

\author{
Cristina Espinoza Moreira / Marcelo Vacacela Álvarez
}

\section{RESUMEN}

El objetivo principal de esta investigación fue establecer la prevalencia de depresión en 90 adultos mayores de 75 años sin deterioro cognitivo severo ni dependencia total que pertenecen a un centro gerontológico. Para ello se aplicaron las pruebas Escala de Depresión Geriátrica de Yesavage (GDS-4) e Inventario de depresión de Beck (BDI-II) en 74 mujeres y 16 hombres; además, una prueba sobre el estado Socio-Familiar. En la primera prueba, el $79 \%$ no presentó manifestaciones depresivas, el indicador depresivo más común fue Problemas de Memoria; en la segunda prueba, la puntuación promedio general fue de 11.36 (entre 0-63), el $71 \%$ No presenta depresión, el 13\% tiene un nivel Leve, el 10\% un nivel Moderado y el $5 \%$ un nivel Severo, se destacan las escalas de Pérdida de interés en el sexo, Agitación, Autocrítica, Cambios en los hábitos de sueño, Dificultad de concentración, Pérdida de energía, y llanto como indicadores de depresión. En la tercera prueba, la mayoría muestra una Buena adaptación social en general y al presentarse indicadores depresivos un aumento del Riesgo Social. Se concluye que en esta muestra la mayoría de los adultos mayores no presentan depresión. Se recomienda en futuros estudios el uso de grupos de control.

\section{PALABRAS CLAVE}

Depresión, adulto major; GDS-4, BDI-II, Guayaquil, vejez, centro gerontológico.

\begin{abstract}
The main objective of this research was to establish the prevalence of depression in 90 adults older than 75 years, without severe cognitive impairment and total dependence belonging to a geriatric center. To fulfill this purpose the Geriatric Depression Scale (GDS- 4), the Beck Depression Inventory (BDI- II) and a social and family status scale were used to tests 74 women and 16 men. In the first test, $79 \%$ had no depressive manifestations, the most common indicator of depression was memory problems; in the second test the overall average score was 11.36 (on a $0-63$ scale), $71 \%$ didn't have depression, $13 \%$ have a Nild level, $10 \%$ a Moderate level and the 5\% a Severe level, the most common depression indicators were Loss of interest in sex, Agitation, Self-Criticalness, Changes in sleep pattern, Concentration difficulty, Loss of energy and Crying. In the third test, most of the sample showed good social adjustment and an increased social risk as depressive indicators appeared. We conclude that in this sample the majority of elders do not have depression. The use of control groups is recommended in future studies.
\end{abstract}

\section{KEYWORDS}

Depression, elder; GDS-4, BDI-II, Guaraquil, aging, geriatric center: 


\section{Introducción}

Los adultos mayores atraviesan por una serie de procesos en el envejecimiento, los cuales dependen de diversos factores que influirán en su calidad de vida. Dentro de esta población van a existir diferentes respuestas, ya sean favorables o inadecuadas, frente a estos cambios. La depresión es uno de los trastornos mentales de mayor complejidad, ya que en el adulto mayor tiene una estrecha relación con factores propios de esta edad, como la dependencia, el deterioro de las funciones cognitivas y el bienestar (Nance, 2010); además de los costos económicos y dificultades en el cuidado del adulto mayor deprimido (Ladin, 2012). En Ecuador, de acuerdo a la Encuesta de Salud, Bienestar y Envejecimiento (SABE, 2009-2010) el 39\% de los adultos mayores de 75 años tienen depresión leve o moderada.

Debido a la manifestación de enfermedades crónicas en esta edad, escasos recursos y pobre participación social, se considera a los adultos mayores como grupo poblacional de atención prioritaria (Ministerio de Inclusión Económica y Social [MIES], 2012). Sin embargo, este sector es heterogéneo $y$ la existencia de vulnerabilidad de cada individuo no responde únicamente a su edad cronológica (Araníbar, 2001). Por lo tanto los adultos mayores, así como otros sectores poblacionales, son influidos por factores bio-psico-sociales, con sus respectivas características que hacen único a cada grupo.

A partir del fácil acceso al estudio de adultos mayores asilados u hospitalizados y a políticas de acogida al "anciano" con características de dependencia, pobreza, maltrato y marginación social, se ha dejado de lado al adulto mayor perteneciente a comunidades de atención integral y esparcimiento, que permanece independiente, activo, que goza de una relativa buena salud y que además están desligados de recibir únicamente una ayuda unidireccional institucional.
Esta investigación se centró en la población descrita para establecer la prevalencia de depresión en el adulto mayor que acudió al Centro Municipal Gerontológico "Dr. $\lambda$ rsenio de la Torre Marcillo" y al IEES en la ciudad de Guayaquil, durante los meses de mayo a agosto del 2013. Para ello se estableció la existencia de manifestaciones depresivas mediante la Escala de Depresión Geriátrica de Yesavage de cuatro ítems, y además se obtuvo los niveles de depresión mediante el Inventario de Depresión de Beck (BDIII). Se realizó además una comparación entre las pruebas de depresión y el estado SocioFamiliar, obtenido de la escala de Valoración Clínica del Adulto Mayor (VACUM) y los datos demográficos.

\section{Marco teórico}

Generalidades y factores de influencia en el adulto mayor

Se consideran adultos mayores las personas que sobrepasan los 65 años. Sin embargo el proceso de envejecimiento es individual y responde a cambios físicos, psicológicos y sociales. Dentro de los cambios físicos, Robert S. Feldman en su libro "Desarrollo psicológico a través de la vida" (2008) establece una distinción entre el envejecimiento primario y secundario. Los cambios que se muestran en el primer tipo se relacionan con la inevitable degeneración funcional, impulsada por las alteraciones genéticas normales (Feldman, 2007, pág. 595). Esto incluye disminución en la velocidad y eficiencia en la respuesta a los estímulos (Dörr, 2008, pág. 373), reducción de la estatura y de la eficiencia de los sistemas corporales, deterioro de la agudeza de los sentidos, baja capacidad respiratoria, afecciones cardiovasculares, problemas renales, entre otros (Fernández, González, \& González, 2006, pág. 471).

La segunda categoría, envejecimiento secundario, se diferencia por la influencia directa del estilo de vida, del contexto cultural 
y social. Se expresa a través de enfermedades crónicas y degenerativas que conducen al deterioro funcional $y$ a la dependencia. De acuerdo al MIES, en el Ecuador, las más comunes en el adulto mayor son: "enfermedades cardíacas, cáncer, enfermedades cerebro vasculares, enfermedades pulmonares, diabetes, hipertensión arterial, deterioro cognitivo y depresión." (MIFS, 2012). Como en toda etapa vital, el nivel de actividad física y la calidad de la alimentación van a tener una fuerte influencia sobre la salud.

Entre los cambios sociales del adulto mayor se encuentra la jubilación. Como consecuencia de este fenómeno, los ingresos disminuyen o se mantienen fijos, porque no responden a los cambios de la economía social, como la inflación. Esto convierte al adulto mayor en una parte vulnerable de la población, ya que, además del costo de la vida, se suma el aumento del cuidado de la salud (Feldman, 2007). El cese de las actividades laborales, obligatorio en muchos casos, impulsa al adulto mayor a tomar un nuevo lugar dentro de la sociedad y de la economía, y su adaptación al nuevo rol relacionado con el ocio y el tiempo libre (Fernández et al., 2006, pág. 479).

Es indispensable destacar la importancia de las redes sociales en el adulto mayor. De acuerdo a Zulma Santos (2009) la integración social ayuda a "mantener la percepción y el sentido de control sobre las situaciones, lo que disminuye los riesgos de morbilidad, mortalidad e internamiento de los adultos mayores." (Santos, 2009). Agrega que la capacidad del individuo de afrontar los cambios que sobrevienen en la vejez estará relacionada con la interacción con sus sistemas familiares y sociales.

En referencia a las relaciones interpersonales, ocurren cambios característicos en esta etapa de la vida. Dentro del matrimonio, sobrevienen eventos como la nueva cercanía derivada de la jubilación, el cuiclado del cónyuge que envejece o la posible viudez. Ia familia es la principal red a la que el adulto mayor pertenece. En muchas familias se les ofrece el rol de consejero de los miembros, en especial sobre la crianza de los nietos (Feldman, 2007). En algunos casos, incluso el cuidado de los nietos desciende parcialmente sobre los abuelos (Fernández et al., 2006). I La amistad durante la adultez tardía toma un matiz de empatía y afrontamiento frente a los fenómenos normales de la vejez (Feldman, 2007, pág. 649). Estos factores antes mencionados, al estar interrelacionados, van a tener una gran importancia sobre el desarrollo psicológico en la adultez tardía. Y de igual manera las características individuales influirán sobre los aspectos físicos y sociales.

Otto Dörr menciona que en la tercera edad se produce un cambio en cuanto a la percepción del tiempo y del espacio. Se produciría el alejamiento y la contemplación, la reducción de la movilidad y la coexistencia de lo esencial y lo accesorio. La temporalidad, por su parte, se enlentece por la ausencia de proyectos de gran importancia como los realizados en la adultez (Dörr, 2008).

Ante la disminución de la actividad y la cercanía de la muerte, numerosos autores como Erikson, Peck, Neugarten, Levinson, entre otros, señalan que es común que ocurra un análisis retrospectivo de la vida, para evaluarla y llegar a una mejor comprensión (Feldman, 2007). Hearn, Saulnier, Strayer, Glenham, Koopman y Marcia (2011) realizaron un estudio de validación del constructo "Integridad versus desesperación" desarrollado por Erickson como última etapa del desarrollo psicosocial en la cual agregaron dos subetapas llamadas Pseudointegridad y No-exploración. Las diferencias entre cada etapa se encuentra en los niveles de apertura a nuevas experiencias, madurez social, 
autovaloración, identidad, tolerancia a la ambigüedad y razonamiento moral (Hearn et al., 2011).

Las respuestas del adulto mayor ante los factores revisados son de dirersos tipos. Por ejemplo, la adaptación puede resultar satisfactoria y produciéndose cambios mínimos en la calidad de vida del individuo, sin embargo existe la posibilidad de la aparición de psicopatologías. Dentro de las alteraciones descritas como más comunes en el İcuador (NIES, 2012), tomaremos la depresión.

\section{Depresión}

Según la Organización Mundial de la Salud (OMS) la depresión es un trastorno mental, en el cual se presenta "tristera, pérdida de interés o placer, sentimientos de culpa o falta de autoestima, trastornos del sueño o del apetito, sensación de cansancio y falta de concentración." (Organización Mundial de la Salud, 2014). Se indica además su carácter frecuente de evolución crónico o recurrente, además de afectar la actividad diaria y social. Como referencia internacional, la OMS recomienda el uso de la Clasificación internacional de enfermedades, en su décima edición (CIE-10). En relación a la depresión, esta hace una categorización de los trastornos del humor o afectivos en la que se describe el episodio depresivo, el trastorno depresiro recurrente, los trastornos del humor 0 afectivos persistentes, otros trastornos del humor; y sin especificación (Guía de bolsillo de la clasificación CIE-10, 2010).

A su rez, el Manual diagnóstico y estadístico de los trastornos mentales en su quinta edición (DSM-V, 2013), describe como característica común entre los trastornos depresiros la presencia de ánimo triste, vacío o irritable, acompañado de cambios somáticos y cognitivos que afectan significativamente la capacidad del funcionamiento individual (American Psychiatric Association, 2013).
Ambas definiciones señalan elementos claros de diagnóstico de la depresión que incluyen aspectos cognitivos, somáticos, afectivos y conductuales.

El modelo cognitivo basa su teoría sobre la depresión en el modelo de diátesis-estrés, el cual indica que factores de rulnerabilidad en el individuo se activan por situaciones estresantes. Como factor primordial se señalan las experiencias tempranas que desarrollarán el escuema cognitivo, subdividido a su ve\% en sesgos de memoria, atención, interpretación, atribuciones causales, pensamientos irracionales, entre otros (V'ázquez, Hervás, Hernangómez, \& Romero, 2010). Estos esquemas disfuncionales van a influir negativamente sobre la persona, el mundo y el futuro (triada cognitiva), desencadenando asi la depresión (Beck, 1967, 1976, citado en "MIodelos cognitivos de la depresión: L'na síntesis y nueva propuesta basada en 30 años de investigación", 2010). $\lambda$ este modelo lineal Vázcuez et al. (2010) lo re-teorizaron como un ciclo. Se presenta de esta manera: luego del esquema cognitivo sigue el estado de ánimo disfórico, los modos de procesamiento (rumiaciones) con estrategias de supresión de pensamientos, que afectarán la tríada cognitiva y; a su rez, derivarán en depresión. Como último elemento se adiciona el estrés, que potencia la repetición.

\section{Depresión en el adulto mayor}

Se establece una diferencia entre la pseudodemencia depresiva y la demencia. En la primera el déficit neurológico se encuentra ausente, existe lentificación, fallas leves de la atención, concentración, memoria yo orientación. Además se presenta desánimo y tristeza con poca reactividad que algunas veces, se encuentra relacionada con un acontecimiento específico, a diferencia de la demencia que aparece de forma gradual (Retamal, 2008). 
De acuerdo a Hernández, Soto y Castro (2010) las manifestaciones de la depresión en el adulto mayor podrian expresarse a través de quejas somáticas exageradas (Bryła, Burzyńska, \& Manieka-Bryła, 2013) (Sociedad Española de Psicogeriatría, 2009), cansancio excesivo, ansiedad $e$ inquietud. En el área del pensamiento podrían presentarse "ideas de indignidad, minusvalia, culpa, desamparo, falta de sentido en la vida e ideas suicidas" (Hernández, Soto, \& Castro, 2010). Otra diferencia es descrita por Nlcalá, Camacho y. Giner (2007) al comparar las emociones negativas básicas entre ancianos y' adultos. En la tercera edad se presenta "una menor intensidad de la afectividad negativa, a expensas de las emociones de miedo, hostilidad y culpa" (Alcalá, Camacho, \& Giner, 2007).

Estudios señalan que la comorbilidad de la depresión con trastornos de ansiedad (Saucedo, Mendieta, Váquez, \& Salinas, 2005), el abuso de sustancias, la hipocondría y el delirio (Weil, 2008), dificultan su diagnóstico, el éxito de la intervención y su recuperación (Pérez \& $\lambda$ rcia, 2008). Estos factores problematizan el abordaje adecuado de la depresión en el adulto mayor.

En algunos casos es indispensable la hospitalización o el ingreso en instituciones $y$ asilos debido a la dependencia total o enfermedades con manifestaciones agudas. Es en estas poblaciones donde se refleja la alta incidencia de depresión (ElKady \& Ibrahim, 2013) (Natínez, Martínez, Esquivel, \& Velasco, 2005). Otra de las dificultades en el abordaje de la depresión radica en que muchos de los estudios realizados sobre el adulto mayor se restringen a personas hospitalizadas, institucionalizadas, asiladas, o los grupos de control son individuos que acuden a centros de salud pública como pacientes o familiares, por lo que se presenta la posibilidad de variables no controladas. Este tipo de muestra no refleja al sector poblacional que se mantiene relativamente activo durante la rejez.

\section{$\mathrm{E}$ l adulto mayor insertado en la comu-} nidad: Redes socio-familiares

Un estudio comparatio entre adultos mayores institucionalizados y no institucionalizados reportó que los primeros muestran una menor autoeficacia y redes sociales más pequenas. (Godoy-Izquierdo, I ara, Vázquez, Araque, \& Godoy, 2012). Además, factores como una menor edad, mejor salud, estabilidad en las relaciones familiares y de amistad pueden influir en la disminución de la depresión en adultos mayores (Horowitz, Reinhardt, Boerner, \& Travis, 2003) o en su baja incidencia (Castro, Ramirez, Aguilar, \& Díaz, 2006).

Igualmente, los adultos mayores que han desarrollado más los aspectos familiares, laborales $y$ emocionales, son quienes se sienten más conformes con su vida actual (Herrera, Romero, Gonzáles, \& Guillén, 2008) y tienen menor riesgo de presentar depresión (RubioAranda, y otros, 2012). Ia actividad física también se incluye entre los factores protectores en la vejez. Durán, ( )bregoz, Uribe y Uribe (2007) concluyeron que "la autonomia y las habilidades funcionales del adulto mayor están estrechamente relacionadas con la frecuencia de las actividades físicas $y$ de integración social."(Durán, ()bregoz, Uribe, \& Uribe, 2008).

\section{Metodología}

La investigación es descriptivo simple, correlacional, transversal, con un enfoque cuantitativo (Gómez, 2009). Los datos usados en esta investigación son parte del proyecto "Identificación de los factores que inciden en la longevidad en la población adulta mayor de 75 años de la ciudad de Guayaquil".

La población comprende adultos mayores de 75 años, activos, independientes y con ca- 
pacidad de movilización, sin deterioro cognitivo o en niveles que le permitan participar en entrevistas, con enfermedades crónicas controladas o sin enfermedades, de la ciudad de Guayaquil. La muestra inicial fue de 117 personas que asistian al Centro Gerontológico "Dr. Arsenio de la Torre Marcillo" y al IEES durante los meses de mayo a agosto del 2013. Fueron escogidas aleatoriamente, controlando únicamente los parámetros de inclusión. Seis personas no cumplieron con el criterio de inclusión de edad, nueve personas no concluyeron la prueba, a ocho no se los pudo ubicar, dos faltaron por enfermedad y dos se encontraban de viaje clurante la recolección de datos; por lo tanto, la muestra final fue de 90 personas.

Los datos fueron recogidos dentro de las instalaciones del centro gerontológico $y$ en auditorios del IEES, por estudiantes de psicología clínica, previamente preparados en la toma de las pruebas previstas. Se citó a los participantes y se les explicó el estudio, al que accedieron voluntariamente. Luego se procedió individualmente a la recolección de datos demográficos usando una ficha técnica que incluyó nombre, edad, sexo, estado civil y familiar responsable.

De forma oral se enunciaron los 4 ítems de la Escala de Depresión Geriátrica de Yesavage (GDS-4), tomada de la Valoración Clínica del Adulto Mayor (VACUM) que incluye la valoración funcional (Katz), valoración cognitiva (Pfeiffer) y la valoración Socio-Familiar. La primera prueba consiste en preguntas de Sí o No, y mide la existencia de síntomas cognitivos de un episodio depresivo mayor en adultos mayores. Los ítems incluyen temas como la Satisfacción con la vida, Impotencia o indefensión, Problemas de memoria y Abulia. Se califica con un punto por cada respuesta que indique un síntoma depresivo. El punto de corte es de 2, su aparición es indicador de manifestaciones de- presivas. $\Lambda$ demás se administró la Escala de Funcionamiento Socio-familiar. Esta escala consta de 10 ítems con dos posibilidades de elección que describen la situación familiar ${ }^{\prime}$ las relaciones sociales. Ia valoración del funcionamiento Socio-Familiar se indica como Buena, Riesgo social y Problema social.

Después se aplicó la segunda edición del Inventario de Depresión de Beck (BDI-II) en su adaptación argentina por Brenlla y Rodríguez (2006) (Beck, Steer, \& Brown, 2006), la cual evalúa los síntomas del trastorno depresivo mayor en adolescentes y adultos. Consta de 21 ítems que describen elementos cognitivos, somáticos y anímicos de la depresión en un lapso de dos semanas, hasta el día de la prueba. Cada opción se divide en grados de intensidad; No presenta (0), Leve (1), Moderado (2) y Grave (3). Se suman las puntuaciones y se ubica por nivel. Los puntos de corte utilizados son: $0-13$ No presenta depresión, 14-19 Leve, 20-28 Moderado, 2963 Severo. El análisis de los datos emplea un método deductivo, descriptivo, correlacional, a través de tablas cruzadas.

\section{Análisis de resultados \\ Datos demográficos}

De los 90 participantes, la edad promedio fue de 80,17 años, con una desviación estándar de 4,5; el $82 \%$ fueron mujeres, $18 \%$ hombres; el $48 \%$ del total de la muestra era viudo, el $30 \%$ casado, $17 \%$ era soltero, $3 \%$ en unión libre y el $2 \%$ divorciado. Entre los familiares con los que conviven los participantes del estudio, el $38 \%$ vive solo con un hijo, el $19 \%$ vire solo, el $18 \%$ vive con el cónyuge, el $10 \%$ vive con su familia, el $9 \%$ con familia extendida (sobrinos, nietos), el 3\% con un hermano y el 3\% con un cuidador. En el Estado Socio-Familiar, el $84 \%$ presenta una Buena adaptación, mientras que el $16 \%$ se encuentra en Riesgo Social. 


\section{Pruebas de depresión}

\section{GDS-4}

En cuanto a los resultados de la escala GDS-4, en el $79 \%$ no se encontraron manifestaciones depresivas, mientras que el $21 \%$ presenta síntomas depresivos. En cuanto a los resultados por ítem, el 96\% del total de la muestra indicó sentirse satisfecho con su vida, mientras que el $4 \%$ no se siente así; el $25 \%$ indica sentirse impotente o indefenso, el $75 \%$ señala que no; el $31 \%$ responde que sí tiene problemas de memoria, mientras que el $69 \%$ no las tiene; y el 16\% señala tener abulia, mientras que el $84 \%$ responde que no en este ítem. En la siguiente tabla se muestran los resultados por ítem divididos por diagnóstico:
En relación a los datos demográficos, el promedio de edad en la muestra sin manifestaciones depresivas es de 80,74 años, con una edad mínima de 75 y la máxima de 97 y una desviación estándar de 4,88. En cuanto a las personas con manifestaciones depre sivas, el promedio de edad es de 78,05, con una edad mínima de 78 y una máxima de 83 , además la desviación estándar es de 2,36. Se distingue la heterogeneidad en cuanto a edad en la muestra sin manifestaciones depresivas, sin embargo, en el grupo con manifestaciones depresivas se destaca su menor edad, en relación al conjunto total de la muestra. En la siguiente tabla se muestran los datos de sexo y estado civil, divididos por el diagnóstico (ver Tabla 2).

Se observa que la mayoría de las mujeres no presentan manifestaciones depresivas, con un $80 \%$ del total de mujeres; en los hombres se observa algo similar, con el $75 \%$ del total de hombres sin manifestaciones depresivas. En la división del estado civil por diag-

Se observa que, en general, más de la mitad de la muestra no presenta síntomas depresivos, sin embargo, el porcentaje más alto de síntoma depresivo es el que corresponde a problemas de memoria, seguido por impotencia o indefensión. Dentro de la muestra sin manifestaciones depresivas, destaca que todos se sientan satisfechos con la vida. En las personas con manifestaciones depresivas, los síntomas que superan a la mitad de la muestra son los Problemas de memoria y la Impotencia e indefensión.

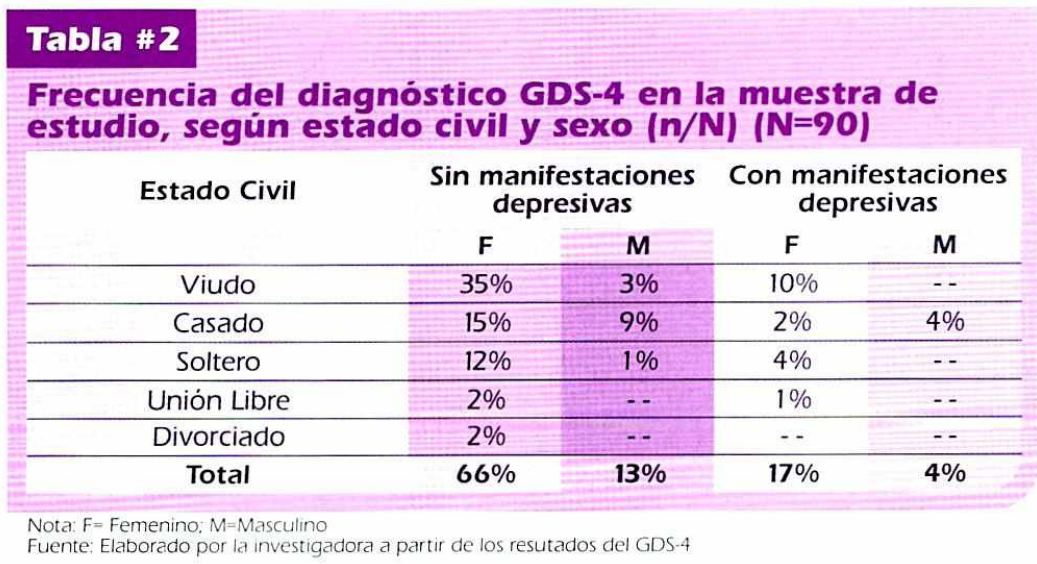

101 
En la siguiente tabla se muestran los resultados del diagnóstico de GDS-4 por los familiares con los que convive el adulto mayor y sexo:

\section{Tabla \#3}

Frecuencia del diagnóstico GDS-4 en la muestra de estudio, según convivencia y sexo ( $n / N)(N=90)$

\begin{tabular}{|c|c|c|c|c|}
\hline \multirow[t]{2}{*}{ Convivencia } & \multicolumn{2}{|c|}{$\begin{array}{c}\text { Sin manifestaciones } \\
\text { depresivas }\end{array}$} & \multicolumn{2}{|c|}{$\begin{array}{c}\text { Con manifestaciones } \\
\text { depresivas }\end{array}$} \\
\hline & $\mathrm{F}$ & M & $F$ & $\mathrm{M}$ \\
\hline Hijo(a) & $27 \%$ & $4 \%$ & $6 \%$ & $1 \%$ \\
\hline Solo & $11 \%$ & $1 \%$ & $7 \%$ & 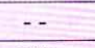 \\
\hline Cónyuge & $7 \%$ & $8 \%$ & $1 \%$ & $2 \%$ \\
\hline Familia & $8 \%$ & -. & $1 \%$ & $1 \%$ \\
\hline Hermano & $2 \%$ & -. & $1 \%$ & -- \\
\hline Cuidador & $3 \%$ & $\cdots$ & $\cdots$ & $\cdots$ \\
\hline Otros (Sobrinos, nietos) & $8 \%$ & $\ldots$ & $1 \%$ & -- \\
\hline Total & $66 \%$ & $13 \%$ & $17 \%$ & $4 \%$ \\
\hline
\end{tabular}

ticos de la prueba GDS-4, sin embargo el porcentaje de Riesgo Social aumenta con la aparición de manifestaciones depresivas.

\section{BDI-II}

En la prueba BDI-II la puntuación promedio general fue de 11.36, con una desviación estándar de 8,23 , lo cual corresponde al nivel de puntuación de No presenta depresión. En relación a los niveles dentro de la muestra, el $71 \%$ No presenta depresión, con 6,9 como puntaje promedio y una desviación están-

Se observa que ningún porcentaje en la convivencia sobrepasa la mitad de la muestra, sin embargo se observa que la mayoría de mujeres sin manifestaciones depresivas viven dar de 3,31; mientras que el 13\% tiene un nivel Leve, con un puntaje promedio de 17,16 y una desviación estándar del 1,33; el 10\% presenta un nivel Moderado con un promecon un hijo, mientras que la mayoría de hombres sin manifestaciones depresivas viven solamente con el cónyuge.

De acuerdo al estado Socio-Familiar, en las personas sin manifes taciones depresivas, el $92 \%$ presenta una buena adaptación, mientras que el $8 \%$ presenta Riesgo social. En cuanto a las personas con manifestaciones depresivas, el 69\% tiene un estado SocioFamiliar bueno, mientras que el $31 \%$ presenta Riesgo Social. Se observa entonces que más de la mitad de la muestra presenta buena adaptación dentro de los diagnós-

\begin{tabular}{|c|c|c|c|c|}
\hline \multicolumn{5}{|c|}{ Frecuencia de respuestas de BDI-Il por escalas $(\mathbf{n} / \mathbf{N})(\mathbf{N}=20$} \\
\hline \multirow[b]{2}{*}{ Escala } & \multicolumn{4}{|c|}{ Grado de respuesta } \\
\hline & 0 & 1 & 2 & 3 \\
\hline Tristeza & $83 \%$ & $17 \%$ & $\cdots$ & $-\cdot$ \\
\hline Pesimismo & $88 \%$ & $10 \%$ & $\cdots$ & $2 \%$ \\
\hline Fracaso & $84 \%$ & $9 \%$ & $6 \%$ & $1 \%$ \\
\hline Pérdida de placer & $71 \%$ & $20 \%$ & $7 \%$ & $2 \%$ \\
\hline Sentimientos de culpa & $72 \%$ & $25 \%$ & $1 \%$ & $2 \%$ \\
\hline Sentimientos de castigo & $82 \%$ & $10 \%$ & $4 \%$ & $4 \%$ \\
\hline Disconformidad con uno mismo & $88 \%$ & $8 \%$ & $2 \%$ & $2 \%$ \\
\hline Autocritica & $68 \%$ & $3 \%$ & $28 \%$ & $1 \%$ \\
\hline Pensamientos o deseos suicidas & $98 \%$ & $1 \%$ & $1 \%$ & $\cdots$ \\
\hline Llanto & $64 \%$ & $11 \%$ & $7 \%$ & $18 \%$ \\
\hline Agitación & $60 \%$ & $13 \%$ & $4 \%$ & $23 \%$ \\
\hline Pérdida de interés & $81 \%$ & $11 \%$ & $4 \%$ & $4 \%$ \\
\hline Indecisión & $71 \%$ & $19 \%$ & $6 \%$ & $4 \%$ \\
\hline Desvalorización & $80 \%$ & $10 \%$ & $6 \%$ & $4 \%$ \\
\hline Pérdida de energía & $46 \%$ & $43 \%$ & $9 \%$ & $2 \%$ \\
\hline Cambios en los hábitos de sueño & $28 \%$ & $37 \%$ & $17 \%$ & $18 \%$ \\
\hline a) Aumento & & $9 \%$ & $2 \%$ & $2 \%$ \\
\hline b) Disminución & & $28 \%$ & $15 \%$ & $16 \%$ \\
\hline Irritabilidad & $87 \%$ & $11 \%$ & $1 \%$ & $1 \%$ \\
\hline Cambios en el apetito & $52 \%$ & $32 \%$ & $13 \%$ & $3 \%$ \\
\hline a) Aumento & & $20 \%$ & $7 \%$ & $\cdots$ \\
\hline b) Disminución & & $12 \%$ & $6 \%$ & $3 \%$ \\
\hline Dificultad de concentración & $50 \%$ & $30 \%$ & $16 \%$ & $4 \%$ \\
\hline Cansancio o fatiga & $52 \%$ & $36 \%$ & $8 \%$ & $4 \%$ \\
\hline Pérdida de interés en el sexo & $24 \%$ & $10 \%$ & $6 \%$ & $60 \%$ \\
\hline
\end{tabular}


dio de 24,1 puntos y una desviación estándar de 2,9; y así mismo, de la muestra total, el $5 \%$ presenta un nivel Serero con un puntaje promedio de 31,6 y una desviación estándar de 4,2 .

Los resultados de acuerdo al grado de respuesta y divididos por escala se muestran en la Tabla 4.

Se observa que en general, las respuestas de las escalas se inclinan hacia el grado de respuesta 0 , en el cual no se presentan los síntomas depresivos. Sin embargo, las escalas de Pérdida de energía, Cambios en los hábitos de sueño, Dificultad de concentración y Pérdida de interés en el sexo, indican que menos de la mitad no presentan estos síntomas. Resaltan a su vez los porcentajes superiores a 20 en la escala de Autocrítica en el grado Moderado de respuesta, y en la escala de Agitación en el grado severo. la escala Pérdida de interés en el sexo, cuyo porcentaje es superior a la mitad de la muestra corresponde al grado severo de respuesta.

En la diferenciación por niveles de puntaje, dentro del nivel de diagnóstico No presenta, la mayoría de las escalas muestran el grado de respuesta 0 . Sin embargo, las escalas que no siguen este patrón de distribución, son Cambios en los hábitos de sueño, con el $44 \%$ del total de No presenta con alteraciones leres (grado 1); la escala de $\Lambda$ gitación, con el 14\% corresponde a respuestas de grado 3; y la escala de Pérdida de interés en el sexo, el $55 \%$ en grado 3 .

En los puntajes de nivel de diagnóstico L_eve, la distribución de la muestra se ubica dentro de cada escala entre los grados de respuesta $0 y$ 1. Las escalas con otra distribución son iutocritica con el 58\% con un grado de respuesta 2; la escala de Agitación con el 33\% en grado 3 y la escala de Pérdicla de interés en el sexo con el $58 \%$ en grado 3 .
En cuanto al nivel de puntuación Moderado, se observa una distribución como el nivel leve. I as escalas fuera de este patrón son las de Autocritica, con un 67\% en grado 2 de respuesta, Dificultad concentración con el $67 \%$ en grado 2, Llanto con el $67 \%$ en grado 3 y. Pérdida de interés en el sexo con el $89 \%$ en grado de respuesta 3 .

El último nivel de puntaje, Severo, muestra una distribución con puntajes concentrados en los grados de respuesta $2 y 3$. Se destaca la escala de Pesimismo, con el 100\% de respuestas de grado 0, el 80\% en ausencia de Pensamientos o deseos suicidas, y la escala de Pérdida de interés en el sexo, con el $80 \%$ de respuestas de grado 3. Fl resultado sobre Pesimismo concuerda con lo indicado en el marco teórico según Alcalá, Camacho, \& Giner, 2007, quienes mencionan que en la tercera edad se presenta "una menor intensidad de la afectividad negativa, a expensas de las emociones de miedo, hostilidad y culpa" (ilcalá, Camacho, \& Giner, 2007)

Se observa de esta manera que los marcadores de sintomas depresiros en la muestra se encuentran elerados en las escalas de Pérdida de interés en el sexo, Agitación, Autocrítica, Cambios en los hábitos de sueño, Dificultad de concentración, Pérdida de energía, y I lanto.

En referencia a la edad de los participantes, el promedio en el nirel de puntaje No presenta, es de 80,32 , con una edad mínima de 75 años, una edad máxima de 97 años y una desviación estándar de 4,81; en los puntajes de nivel l.eve, la edad promedio es de 80,25 , con una edad mínima de 75 , una máxima de 93 y una destriación estándar de 5,04; en el nivel Moderado, el promedio de edad es de 79,88, la edad mínima es de 76 años, la edad máxima de 83 y la desriación estándar de 2,71; por último, en el nivel de puntaje Severo, la edad promedio es de 78,6 años, la 
edad mínima es de 75 años, la máxima de 85 años y la desviación estándar de 3,91. Se observa que dentro de la muestra; mientras el ni vel de depresión aumenta, la edad promedio disminuye; sin embargo, ha de notarse que las diferencias son pequeñas. Entre No depresión $(80,32)$ y Leve $(80,25)$, la diferencia es de 0,07. Entre Leve y Moderado $(79,88)$, es de 0,37 . Entre Moderado y Severo $(78,6)$, es de 1,28. Y entre los dos extremos de No depresión y Severo, la diferencia es de 1,04. Por tanto, este dato merece un estudio posterior más detallado, con una muestra más amplia.

\section{En referencia al} sexo, más de la mitad de la muestra dividida por esta variable no presenta depresión. Las mujeres muestran que el $73 \%$ del

\section{Tabla \#6}

Frecuencia de niveles de diagnóstico de BDI-II por sexo y convivencia $(\mathrm{n} / \mathrm{N})(\mathrm{N}=90)$

\begin{tabular}{|c|c|c|c|c|c|c|c|c|}
\hline \multirow[t]{2}{*}{ Convivencia } & \multicolumn{2}{|c|}{ No Presta } & \multicolumn{2}{|c|}{ Leve } & \multicolumn{2}{|c|}{ Moderado } & \multicolumn{2}{|c|}{ Severo } \\
\hline & $\mathbf{F}$ & $M$ & $\mathbf{F}$ & $\mathbf{M}$ & $\mathbf{F}$ & $M$ & $\mathbf{F}$ & $M$ \\
\hline Hijo(a) & $22 \%$ & $6 \%$ & $2 \%$ & -- & $6 \%$ & -- & $2 \%$ & $\cdots$ \\
\hline Solo & $12 \%$ & $\ldots$ & $4 \%$ & $\ldots$ & -- & $1 \%$ & $2 \%$ & -- \\
\hline Cónyuge & $7 \%$ & $4 \%$ & $\cdots$ & $5 \%$ & $\cdots$ & $1 \%$ & $1 \%$ & $\cdots$ \\
\hline Familia & $8 \%$ & $1 \%$ & -- & -- & $1 \%$ & $\ldots$ & -- & -- \\
\hline Hermano & $3 \%$ & $\ldots$ & -- & $\cdots$ & -- & -. & $\cdots$ & $\cdots$ \\
\hline Cuidador & $2 \%$ & -- & $1 \%$ & $\ldots$ & -- & $\ldots$ & -- & $\ldots$ \\
\hline Otros & $6 \%$ & .. & $2 \%$ & -- & $1 \%$ & $\cdots$ & $\ldots$ & $\ldots$ \\
\hline Total & $60 \%$ & $11 \%$ & $9 \%$ & $5 \%$ & $8 \%$ & $2 \%$ & $5 \%$ & $0 \%$ \\
\hline
\end{tabular}

Nota: $F=$ Femenino: $M=$ Masculino
Fuente: Elaborado por la investigadora a partir de los resutados del BDI-II total de mujeres no pre-

sentan depresión, el 11\% tiene un nivel Leve, el 9\% en forma Moderada y el 7\% en un nivel Severo. En los hombres, el 63\% del total de hombres no presenta depresión, el 25\% de forma Leve y el $13 \%$ en un nivel Moderado; no hay hombres con puntaje Severo dentro de la muestra.

En la siguiente tabla se muestran los resultados por niveles de puntuación, sexo y estado civil:

\section{Tabla \#5}

Frecuencia de niveles de diagnóstico de BDI-II por sexo y estado civil $(\mathbf{n} / \mathbf{N})(\mathbf{N}=\mathbf{9 0})$

\begin{tabular}{|c|c|c|c|c|c|c|c|c|}
\hline \multirow[t]{2}{*}{ Estado Civil } & \multicolumn{2}{|c|}{ No Presta } & \multicolumn{2}{|c|}{ Leve } & \multicolumn{2}{|c|}{ Moderado } & \multicolumn{2}{|c|}{ Severo } \\
\hline & $\mathbf{F}$ & $M$ & $\mathbf{F}$ & $M$ & $\mathbf{F}$ & $M$ & $\mathbf{F}$ & $\mathbf{M}$ \\
\hline Viudo & $32 \%$ & $3 \%$ & $5 \%$ & $-\cdot$ & $7 \%$ & $\ldots$ & $1 \%$ & -. \\
\hline Casado & $15 \%$ & $8 \%$ & $1 \%$ & $5 \%$ & $1 \%$ & $1 \%$ & -- & -- \\
\hline Soltero & $10 \%$ & $\ldots$ & $3 \%$ & $\ldots$ & $\cdots$ & $1 \%$ & $2 \%$ & -- \\
\hline Unión Libre & $1 \%$ & $\ldots$ & $\ldots$ & $\ldots$ & -. & -. & $2 \%$ & $\ldots$ \\
\hline Divorciado & $2 \%$ & $\cdots$ & -- & -. & $\ldots$ & $\cdots$ & -. & $\ldots$ \\
\hline Total & $60 \%$ & $11 \%$ & $9 \%$ & $5 \%$ & $8 \%$ & $2 \%$ & $5 \%$ & $0 \%$ \\
\hline
\end{tabular}

Los resultados de acuerdo a la convivencia no muestran porcentajes mayores a la mitad de la muestra. Dentro del nivel No presenta, el porcentaje más alto en ambos sexos se encuentra en las personas que viven solamente con un hijo; en las mujeres este porcentaje también se encuentra elevado en las que tienen un nivel Moderado de depresión. Además, los hombres que tienen un nivel Leve de depresión viven con el cónyuge.

En comparación con el estado Socio-Familiar, del grupo que No presenta depresión, el $88 \%$ tiene una Buena adaptación social, mientras que el 22\% está en Riesgo Social. Así mismo, de los adultos mayores con puntaje Leve, el $75 \%$ presenta Buena adaptación, 
mientras que el $25^{\circ}$ \% se encuentra en Riesgo Social. En cuanto a las personas con puntaje Moderado, el 89\% tienen Buena adaptación, mientras que el 11\% muestran Riesgo Social. Finalmente, de la muestra con puntaje severo, el 60\% tiene Buena adaptación, mientras que en el $40 \%$ se observa Riesgo Social. Se observa que sin importar el nivel de depresión la muestra presenta una Buena adaptación social. Sin embargo el Riesgo Social se eleva en el nivel Severo de depresión.

\section{Discusión}

$\Lambda$ diferencia de los resultados de la Encuesta de Salud, Bienestar y Envejecimiento (SABE, 2009-2010) que afirman que en Ecuador, el 39\% de los adultos mayores de 75 años tienen depresión leve o moderada, en la muestra de este estudio, los porcentajes de depresión son menores: en el GDS-4, el $21 \%$ y en BDI-II, el $29 \%$. Se observa el $17 \%$ de diferencia con los datos generales ecuatorianos, es por ello que esta muestra, debido a sus características, necesita de mayores estudios.

En relación con el indicador depresivo del GDS-4 Problemas de memoria, no es posible establecer una relación directa con la depresión ya que se cuestiona el papel de los problemas de memoria en la valoración real de un episodio depresivo, o si por su parte corresponde al deterioro cognitivo y los síndromes relacionados (Weil, 2008) Retamal, 2008).

Es importante señalar que se cuestiona la validez en la detección de un episodio depresivo en los adultos mayores de algunas escalas de la prueba BDI. Gonzáles-Celis (2009) en el artículo "Composición factorial del inventario de depresión de Beck en ancianos mexicanos", recomienda eliminar las escalas 19 (Pérdida de peso) y 21 (Pérdida de la libido) de la versión anterior del BDI, debido a su relación con trastornos somáticos, y la escala 14 (Autoimagen negativa) ya que está asociada a prejuicios negativos en la vejez. (González-Celis, 2009). la escala Pérdida del interés en el sexo, tomando en cuenta su inesperado resultado que muestra a más de la mitad sin un interés en el sexo, podría estar relacionado con el desarrollo normal del adulto mayor (Weil, 2008). Sin embargo, al considerarse como característica en la depresión del adulto mayor la aparición de quejas somáticas (Brỵła et al., 2013) (Hernández et al., 2010) (Sociedad Española de Psicogeriatría, 2009), no pueden ser eliminadas en la valoración clínica para el diagnóstico de la depresión. La escala Agitación y Cambios en los hábitos de sueño podrían a su vez relacionarse además del episodio depresiro, con un trastorno de ansiedad (Agudelo, BuelaCasal, \& Spielberger, 2007). De esta manera se hace evidente la dificultad en el diagnóstico de la depresión (entre otros trastornos) en el adulto mayor, además de la necesidad de una teoría unificada y que también aporte con instrumentos válidos de diagnóstico que permitan examinar con mayor precisión muestras como las de esta investigación.

\section{Conclusiones y recomendaciones}

De acuerdo a las pruebas aplicadas, se establece que más de la mitad de los adultos mayores de la muestra no presentan depresión. En la prueba GDS-4 la mayoría señala sentirse satisfecho con la vida, aun cuando tienen manifestaciones depresiras; el indicador más común de depresión son los problemas de memoria, punto que fue cuestionado en la discusión. En la prueba BDI-II, las escalas de Pérdida de interés en el sexo, Agitación, Autocrítica, Cambios en los hábitos de sueño, Dificultad de concentración, Pérdida de energía y Llanto son marcadores de síntomas depresivos.

En las dos pruebas el promedio de edad disminuye mientras aumentan las manifestaciones depresivas y sus niveles. En compara- 
ción con otro estado civil, la mayoría de los hombres casados presentaban indicadores de depresión; sin embargo, al constar una muestra pequeña masculina debe revisar se esta conclusión. En cuanto a la convivencia, la mayoría de las personas que no obtuvieron indicadores depresivos viven con un hijo. Además los hombres que no presentan manifestaciones depresivas (GDS-4) y tienen un nivel leve de depresión (BDI-II) conviven sólo con el cónyuge. No se encontraron otros patrones relacionados con los datos demográficos y los indicadores de depresión.

En cuanto al estado socio familiar, en ambas pruebas la mayoría de la muestra presentó una Buena adaptación social, aun en la aparición de indicadores depresivos. Sin embargo, en el grupo con indicadores depresivos se presentó un aumento del Riesgo Social. En la muestra, el Riesgo Social se eleva en el nivel Severo de depresión. En general, los resultados de este estudio concuerdan con lo expuesto en el marco teórico sobre la importancia que tiene la inserción del adulto mavor en la comunidad, en las redes sociofamiliares.

Al ser una de las limitaciones de esta investigación, en próximos estudios se recomienda la utilización de una entrevista clínica u otros instrumentos que evalúen la incidencia de otros trastornos mentales relacionados con la depresión, como los trastornos de ansiedad, trastornos de somatización, las fobias y episodios psicóticos, para poder establecer con mayor precisión la existencia de trastornos depresivos o su comorbilidad. Otra recomendación se relaciona con la comparación de los indicadores depresiros con las patologías médicas presentes de manera crónica en los adultos mayores, para de esta manera poder formar correlaciones fiables con los diferentes factores de influencia. Además sería de gran importancia poder establecer la prevalencia de depresión y las características de los adultos mayores en general de Guayaquil, o a su vez establecer grupos de control con otro tipo de muestras, ya sean institucionalizadas, asiladas o al azar.

\section{Bibliografía}

Agudelo, D., Buela-Casal, G., \& Spielberger, C. (2007). Ansiedad y Depresión: El problema de la diferenciación a través de los síntomas. Salud Mental, 30(2), 33-41. Recuperado de http://www:medigraphic. $\mathrm{com} / \mathrm{pdfs} / \mathrm{salmen} / \mathrm{sam}-2007 / \mathrm{sam} 072 \mathrm{e}$. pdf

Alcalá, V., Camacho, M., \& Giner, J. (2007). $\Lambda$ fectos y depresión en la tercera edad. Psicotbema, 19(1), 49-56. Recuperado de http://www:psicothema.com/pdf/3327. pdf

American Psychiatric Association. (2013). Depressive Disorders. En American Psychiatric Association, Diagnostic and Statistical Manual of Mental Disorders, Fiffth Edition (pp. 155-188). Arlington: American Psychiatric Association.

Araníbar, P. (2001). Acercamiento conceptual a la situación del adulto major en América Latina. Serie Población desarrollo 21. Recuperado del sitio de Internet de Publicaciones para la Comisión Económica para América Iatina y el Caribe: http://wwwcepal.org/ publicaciones/xml/0/9260/lcl1656p.pdf

Beck, A., Steer, R., \& Brown, G. (2006).BDIII, Inventario de Depresión de Beck, Segunda Edición Manual. Buenos Aires: Paidós.

Bryła, MI., Burzyńska, M., \& Manieka-Brỵła, I. (2013). Self-rated quality of life of citydwelling elderly people benefitting from social help: Results from a cross-sectional study:Health and Quality of Life Ontcomes, 181. doi:10.1186/1477-7525-11-181 
Castro, M., Ramirez, S., Aguilar, L., \& Díaz, V. (2006). Factores de riesgo asociados a la depresión del adulto mayor: Relista Nemrologia, Nemrocingia .) Psiquiatria, 39(4), 132-137. Recuperado de http://www.imbiomed.com/1/1/ articulos.php? method =showDetail\&id_ articulo $=411908$ id_seccion $=80 \&$ id ejemplar $=4214 \&$ \&id_revista $=15$

Dörr, A. (2008). Tercera Edad. En $\lambda$. Dörr, M. E. Gorostegui, \& M. L. Bascunāán (Eds.) Psicologia general y' evolutiva (pp. $370-$ 391). Santiago: Mediterráneo I tda.

Durán, D., Obregoz, L., Uribe, A., \& Uribe, J. (2008). Integración social y habilidades funcionales en adultos mayores. Universitas Psychologica, 7(1), 263-270. Recuperado de http://revistas.javeriana.edu.co/index. php/revPsycho/article/view/213/1691

ElKady, \& Ibrahim. (2013). Depression among a group of elders in Alexandria, Egypt. Eastern Mediterranean Health Joumal, 19(2), 167-174. Recuperado de http://www.ncbi.nlm.nih.gov/pubmed/23516828

Encuesta de Salud, Bienestar y Envejecimiento (SABE). (2010). Encuesta de Salud, Bienestar y Envejecimiento, Resultados. Quito.

Feldman, R. S. (2007). Desarrollo físico y cognoscitivo en la edad adulta tardía. En R. S. Feldman (Ed.)Desarrollo psicológico a través de la vida (pp. 590-621). México: Pearson Educación.

Fernández, M. P., González, E., \&González, M. P. (2006). Los mayores. En E. González (Ed.)Psicologia del ciclo vital (pp. 467498). Madrid: Editorial CCS.
Godoy-Izquierdo, D., Lara, R., Vázquez, M. l., Araque, F., \& Godoy, J. (2012). Correlates of happiness among older spanish institutionalized and non-institutionalized adults. Springer: Science and Bussiness, 389-414. doi: 10.1007/s10902-012$93.35-5$

Gómez, M. (2009). Introducción a la metodología de la investigación científica, Segunda Edición. Córdoba: Editorial Brujas.

González-Celis, A. L. (2009). Composición factorial del inventario de depresión de Beck en ancianos mexicanos. Journal of Belsarior; Health \& Social Issues, 1(1), 15 28. Recuperado de http://www.redalyc. org/articulo.oa?id=282221718002

(2010). Guía de bolsillo de la clasificación CIE-10. Madrid: Editorial Medica Panamericana.

Hearn, S., Saulnier, G., Strayer, J., Glenham, M., Koopman, R., \& Marcia, J. (2011). Between Integrity and Despair: Toward Construct Validation of Erickson's Eighth Stage. Springter Science and Bussiness Media, 19, 1-20.doi: 10.1007/s10804-0119126-y

Hernández, I., Soto, M. E., \& Castro, I. (2010).Envejecimiento Normal y Depresión Clínica en el Adulto Mayor. Santiago, Chile: Universidad Bolivariana.

Herrera, F., Romero, A., Gonzales, E., \& Guillén, L. (2008). Percepción de los ancianos sobre los centros de desarrollo integral para el adulto mayor. Revista IIPSI, 11(1), 129-136. Recuperado de http:// sisbib.unmsm.edu.pe/BVRevistas/Investigacion_Psicologia/v11_n1/pdf/a08. pdf 
Horowit\%, Reinhardt, Boerner, \& Travis. (2003). The infuence of health, social support quality and rehabilitation on depression among disabled elders. Aging and Mental Health, 7(5),342-350.doi: 10.1080/1360786031000150739

J adin, K゙. (2012). Decomposing differences in utilization of health services between depressed and non-depressed elders in Europe. Springer-T'erlag, 9, 51-64. doi: 10.1007/s10433-011-0213-5

Martine\%, J., Martinez, V., Esquirel, C., \& Velasco, V. (2005). Prevalencia de depresión y factores de riesgo en el adulto mayor hospitalizado. Rerista Médica del IMSSS, 45(1), 21-28. Recuperacto de http://revistamedica.imss.gob.mx/index. php?option $=$ com_multicategories\&riew $=$ article\&id=1078:prevalencia-de-depresion-y-factores-de-riesgo-en-el-adultomayor-hospitalizado\&Itemid $=617$

Ninisterio de Inclusión Económica y Social (MIIES), M. d. (2012). Agenda de Igualdad para adultos mayores 2012-2013. Quito: Ministerio de Inclusión Económica y Social (NIIES).

Nance, D. C. (2010). Depresión en adultos mayores. Emrejecinniento bumano: L'ina vision transdisciplinaria.239-248. Recuperado de http://wrux: geriatria.salud.gob.mx/descargas/24.pdf

Organización Mundial de la Salud. (15 de Marzo de 2014). Organización Mundial de la Salud. Obtenido de http://www. who.int/topics/depression/es/

Pérez, V., \& Arcia, N. (2008). Comportamiento de los factores biosociales en la depresión del adulto mayor. Rerista Cubana de Medicina General Integral, 24(3). Recuperado de http://wwww.geriatria.salud. gob.mx/descargas/24.pdf
Retamal, P. (2008). Enfermedades del ánimo. En C. Carrajal, R. Florenzano, \& K. Weil (Eds.) Psiquiatric (pp. 139-156). Santiago: Mediterráneo.

Rubio-Aranda, E., Blanco-Montón, G., Comin-Comín, M., Martinez-Terrer, T., Magallón-Botaya, R., \& García-Campayo, J. (2012). Salud mental y relaciones sociales en población mayor rural. Actas Espaniolas de Psiquiatria, 40(5) 239-247. Recuperado de http://wwwwactaspsiquiatria.es/repositorio/14/79/ESP/14-79ESP-239-247-974920.pdf

Santos, Z. (2009). Adulto mayor, redes sociales e integración. Trabajo social, 159174. Recuperado de http://www.revistas. unal.edu.co/index.php/tsocial/article/ view $/ 15310 / 16103$

Saucedo, D., Mendieta, D., Vásquez, $\Lambda ., \&$ Salinas, R. (2005). Relación entre ansiedad y depresión con quejas somáticas de tipo doloroso en mujeres mayores de 60 años de edad. Medicina Universitaria, 7(26), 123-127. Recuperado de http://content. ebscohost.com/pdf19_22/pdf/2005/ UGL/01Ju105/26308316.pdf?T $=P \&-$ $\mathrm{P}=\mathrm{AN} \& \mathrm{~K}=26308316 \& \mathrm{~S}=\mathrm{R} \& \mathrm{D}=\mathrm{a} 9 \mathrm{~h} \&$ EbscoContent $=\mathrm{dGJ}$ :NNXb4kSeprc4r \%2Bbw()LCmr0yep7BSr664Ta6WxIVX S\&ContentCustomer=dGJyMGnslGuq bRRuePfgerx44Dt6fIA

Sociedad Española de Psicogeriatría. (2009). Consenso Español de depresión en el anciano. Recuperado del sitio de Internet de la Sociedad Española de Psicogeriatría: http://sepg.es/actividades/ConsensoDepresion.pdf

Vázquez, C., Herrás, G., Hernangómez, L., \& Romero, N. (2010). Modelos Cognitiros de la depresión: Una sintesis y nueva propuesta basada en 30 años de investiga- 
ción. Behavioral Psychology; 18(1), 139-165.

Recuperado de http://dialnet.unirioja. es $/$ servlet $/$ articulo? codigo $=3289253$

Weil, K. (2008). Psicogeriatría. En C. Carvajal, R. Florenzano, \& K. Weil (Eds.) Psiquiatria, Segunda edición (pp. 361-372). Santiago: Mediterráneo.

\section{Cristina Espinoza Moreira}

Psicóloga Clínica de la Universidad Espíritu Santo - Ecuador

E-mail: crisespinoza@uees.edu.ec

\section{Marcelo Vacacela Álvarez}

Psicólogo Clínico de la Universidad Estatal de Guayaquil

Magister en Gerencia Educativa

Universidad Espiritu Santo - Ecuador

E-mail: marcevacacela@uees.edu.ec 
